\title{
Research progress in pathogenesis and treatment of neuroinflammation
}

\author{
Yingxin $\mathrm{Shi}^{1}$, Xiangyu Zhang ${ }^{1}$, Heng Zhang ${ }^{1}$, Linfeng $\mathrm{Ge}^{1}$, and Lihui Zhao ${ }^{1, *}$ \\ ${ }^{1}$ Changchun University of Science and Technology, School of Life Science and technology, 130022, China
}

\begin{abstract}
Neuroinflammation, defined as an inflammatory response mediated by central nervous system astrocyte, Microglia and endothelial cells, disrupts homeostasis and is typical of many Neurodegeneration. The DA receptor family is mainly expressed in Microglia, and microglia-induced neuroinflammation is an important pathogenesis of Neurodegeneration, this article reviews the pathogenesis and treatment of neuroinflammation.
\end{abstract}

\section{Introduction}

The maintenance of the normal physiological function and state of the brain is closely related to the fine regulation and protection of glial cells (including Microglia and astrocyte) in the brain, however, the abnormal activation of these two types of glial cells and the release of a variety of inflammatory factors to form a neuroinflammatory response. Whether the brain is naturally aging or central nervous system Degenerative disease, such as Alzheimer's disease, Parkinson's disease. the neuroinflammatory response is widespread and extremely active, leading to immune dysfunction, often accompanied by disease. Finding ways and means to regulate neuroinflammation has important theoretical implications for delaying brain aging and controlling the onset and development of central nervous system Degenerative disease[1]. The study found that Microglia played a leading role in the brain's inflammatory response, while astrocyte played a supporting role, and astrocyte also played a leading role in the development of inflammation in the absence of the dopamine $\mathrm{D} 2$ receptor (DRD2), the levels of dopamine and its ligands showed a decreasing trend in the middle-aged and elderly population[2]. Physiologically, astrocyte DRD2 inhibits the immune response by controlling levels of B-crystallin (Cryab) downstream. The deletion of DRD2 could significantly decrease the level of Cryab and increase the inflammatory response in several regions of mouse brain, while the deletion of DRD2 increased the activation of glial cells in the mouse model of Parkinson's disease induced by the neurotoxin MPTP, the more severe the inflammation, the more sensitive the midbrain dopaminergic neurons to neurotoxins, the higher the death rate. But selectively increasing levels of Cryab in the astrocyte may be effective in combating chronic inflammation caused by DRD2 deficiency. (figure 1)[3]

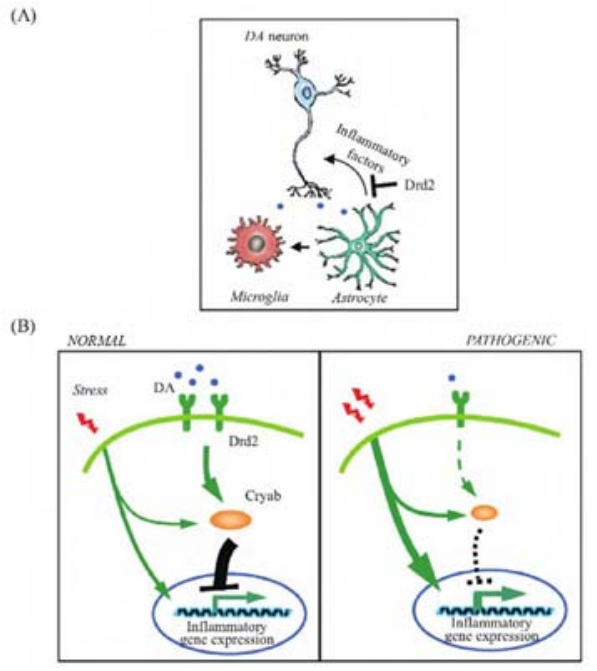

Figure 1. astrocyte Drd2/Cryab signaling pathway plays an anti inflammatory role [3]

A:pattern of communication between astrocyte, Microglia, and neuronal cells; B:under physiological and pathological conditions, the Drd2/Cryab signaling pathway in the astrocyte plays a role in inhibiting the production of inflammatory factors; in aging and age related brain diseases, the DRD2 signaling pathway in the astrocyte is reduced, resulting in reduced Cryab function, which leads to the overproduction of inflammatory mediators. DA: Dopamine.

\section{Inflammation and Neurological Disease}

When the central nervous system is damaged, the astrocyte is in an "active" state, changing its form, number and biological function from benign "resting" to "reactive" astrocytes. In 2012, Barres and his colleagues solved this problem by finding two different types of astrocyte A1 and A2. Inflammation such as bacterial

\footnotetext{
*Corresponding author: 1252042806@qq.com
} 
lipopolysaccharide can induce astrocyte to become harmful type 1 cells, whereas Hypoxia can induce type 2 A2 cells. A2 produces nutrients near ischemic tissue that support the growth of neurons. Activation of the NEUROGLIA can damage neurons. Scientists call these mechanisms neuroinflammation.

Astrocyte are nerve cells that have many important functions in the nervous system. In brain infections and Neurodegeneration, these cells tend to become inflamed, known as astroglitosis. To understand the inflammatory process of astrocyte tumors, Human cells found in the urine of voluntary donors were reprogrammed to become pluripotent stem cells. The authors then stimulated these stem cells to become healthy astrocyte and then subjected them to TNF stimulation. The observed results suggest that the inflammatory process occurs within the first hour after stimulation and gradually weakens the function of the astrocyte over time. In addition to these morphological changes, inflammation interferes with astrocyte's primary function: the regulation of neurotransmitters, the substances that neurons secrete that carry messages through the synapse. The study showed that human astrocyte with astrocyte hyperplasia showed impaired glutamate uptake. It is worth noting that glutamate is the most important source of stimulating neurotransmitters involved in many brain functions, including learning and memory. These studies could improve the quality of life in patients with infections or Neurodegeneration induced inflammation of the brain[4].

The inflammatory response is a double-edged sword. Protective Inflammation helps the body clear away pathogens and tumors. However, prolonged and uncontrolled inflammation can lead to sepsis and many chronic diseases. Acute inflammation is a host-protective, usually self-limiting, response that maintains intracellular homeostasis and promotes defense against a variety of infection and injury factors. However, when poorly managed or activated, acute inflammation can lead to persistent and uncontrolled chronic inflammation, which is associated with many other chronic diseases, including cardiovascular and metabolic diseases[5].Therefore, the inflammatory response needs to be precisely regulated to prevent its overreaction.

The long-term existence of whole brain inflammation may affect the tissue repair after brain injury, continuously destroy the brain structure, inhibit the function of the whole brain, and lead to the delayed pathological changes after stroke, such as dementia, depression and fatigue. The study of neuroinflammation should not only clarify the initiation and maintenance mechanism of whole brain inflammation, but also clarify the possible role of whole brain inflammation in the process of nerve regeneration and repair after brain injury[6].

\section{Dopamine Receptor Mediated Signaling}

\section{1 dopamine and its receptors}

Dopamine (DA) is an organic chemical in the catecholamine and phenylethylamine family that plays several important roles in the brain and body, its name comes from its chemical structure: it is an amine synthesized from its precursor, a molecule called LEVODOPA, which removes carboxyl groups and is found in human brain and adrenal cells. In the brain, dopamine acts as a neurotransmitter, sending signals to other nerve cells through neurons that release chemicals. The brain includes several different dopamine pathways, and dopamine has been found to play an important role in regulating immune inflammation. Several important neurological disorder are associated with dysfunction of the dopamine system, a degenerative condition of Parkinson's that causes tremors and Dyskinesia, it is caused by a deficiency in the production of dopamine by secretory neurons in the midbrain called the substantia Nigra. There is evidence that schizophrenia involves changes in dopamine activity levels, and most commonly used antipsychotic have a reduced dopamine activity effect. The Dopamine Receptor belongs to the G-ProteinCoupled Receptor (GPCR) superfamily of $g$ proteins (consisting of $\mathrm{G}$ and $\mathrm{G}$ subunits) and contains Dopamine Receptor d (Dopamine d) : DRD1, DRD2, DRD3, DRD4, and DRD5. Among them, DRD1 and DRD5 belong to D1 type receptors, which are coupled with activated $g$ protein Gs to stimulate the production of Second Messenger cAMP, while DRD2, DRD3 and DRD4 belong to D2 type receptors, which are coupled with inhibitory g protein $\mathrm{Gi} / \mathrm{o}$ to inhibit the production of Second Messenger cAMP. Among them, dopamine receptor D2(DRD2) is a major target for schizophrenia and Parkinson's disease. Da has been shown to inhibit NLRP3 inflammatory corpuscles and downstream inflammatory responses via DRD1 signaling. However, it has been reported that DA produced by electrical stimulation of vagus nerve can control sepsis by extensively inhibiting the expression of several inflammatory factors including TNF-A, MCP-1, IL-6 and IFN-G through D1 receptors. Therefore, DADRD signaling may have other unknown and complex regulatory mechanisms in addition to regulating inflammation by inhibiting inflammatory bodies. The DRD2 gene is located in the chromosome 11 and is widely distributed in the central nervous system. DRD2 is highly expressed in striatum, nucleus accumbens, Olfactory Bulb, and in dopaminergic neurons of midbrain. The changes of DRD2 in the system are closely related to Parkinson's disease. During the course of aging and Parkinson's disease, the expression of DRD2 is down-regulated. DRD2 knockout mice exhibit Parkinson's-like Bradykinesia symptoms and pathological features. DRD2 agonists have been used as adjuncts to treat Parkinson's disease. There is a lot of evidence that DRD2 plays an important role in the pathogenesis of Parkinson's disease. However, the role of DRD2 on neuroinflammation in Parkinson's disease is not clear. Current data suggest that many neurotransmitter receptors, including Dopamine receptor, are expressed in Microglia and astrocyte, suggesting that classical neurotransmitter signaling pathways regulate the connections between neurons and glial cells. It is not known whether the loss of the Dopamine receptor signal pathway disrupts the homeostasis of the brain by affecting the immune response and communication between the nervous system, 
leading to disease. The concept of Dopamine's own receptor has been around since 1976. It has been found that Dopamine receptor agonists inhibit the release of dopamine, and by using Dopamine receptor specific agonists or inhibitors, DRD2 has been found to be probably the most important dopamine self receptor[7]. The motor function of DRD2 knockout mice was significantly increased, so DRD2 receptor inhibited the release of dopamine and the motor function of mice. The inhibitory effect of DRD2 self-receptors on the axon on the Dopamine endocytosis is of physiological significance, which can limit the action potential induced dopamine overrelease. In patients with Parkinson's disease, the level of DRD2 mRNA in substantia Nigra without degeneration and death dopaminergic neurons increased significantly, which provided new ideas for further elucidating the physiological function and pathological significance of Dopamine receptor in the nervous system and for the development of drug targets[8].

\subsection{Regulation of inflammatory response by Dopamine receptor}

Neuroinflammation is the immune response of the central nervous system (CNS) to various noxious stimuli, such as injury or infection, cNS Neuroglia, endothelial cells and peripheral immune cells mediate and produce cytokines, chemokines, reactive oxygen species and other inflammatory mediators[9].Li found that dopamine and Dopamine receptor in Lipopolysaccharide(LPS) induced peritoneal macrophage-related inflammation in mice, DA through D2 receptor can inhibit LPS-induced TNF- $\alpha$ secretion and thus inhibit TLR4-dependent inflammatory response in mice peritoneal macrophages. Using DRD2 receptor knockout mice and wild type mice, the morphological changes of Cryab in mice and human brain slices were observed, including the injury of dopaminergic neurons, the activation of astrocyte and Microglia, the effects of DRD2 on glial response and neuroinflammation in Parkinson's disease (PD) were studied at cellular and molecular levels, and the results showed that DRD2 could inhibit neuroinflammation by modulating Cryab[10].Microglia, also known as the brain's central nervous system, are innate immune cells that act as the first line of defense when injury or disease occurs. It is primarily responsible for sensing external stimuli, including CNS trauma, Ischemia, infection and the invasion of abnormal pathogens, antigens, toxins and so on, thus causing an immune response and altering the organism's homeostasis. It is important to study the inflammatory response of Microglia to LPS induced neuroinflammation.It was found that the DRD5 receptor of macrophages was activated and the negative regulator ARRB2/ PP2A was recruited into TRAF6-IKK complex by the third inner ring IYX $(X) \mathrm{I} / \mathrm{L}$ structural motif and the end-tail EFD structural Motif, thus blocking the inflammatory signal of NF- $\kappa \mathrm{B}$, to suppress Staphylococcus AUREUS SEPSIS and meningitis. By searching open databases, as well as macrophage Dopamine receptor drugs and gene knock down screening, the researchers found that DA inhibits the inflammatory response of macrophages in the form of NF- $\kappa \mathrm{B}$ via the DRD5 receptor mediated TLR2 pathway. By analyzing all known TLR pathways, it was found that DA mainly inhibited TRAF6-mediated inflammation and did not depend on cAMP-PKA signal downstream of DRD. Subsequently, through protein biochemical experiments, the researchers demonstrated that DRD5 recruits TRAF6 and its negative regulator ARRB2 to form DRD5ARRB2-TRAF6 signal protein complexes via the EFD structural motif and the IYX(X)I/L structural motif of the third intracellular ring, respectively, then ARRB2/PP2A negatively regulates the TRAF6-mediated NF- $\kappa \mathrm{B}$ inflammatory response. Studies have revealed a new neuroimmunomodulatory signaling pathway that first elucidates the molecular mechanism by which macrophage DA-DRD5 signaling inhibits sepsis and meningitis induced by Staphylococcus aureus infection, it also provides a theoretical basis for the development of related anti-inflammatory drugs[11].

\subsection{Signal Transduction Pathways of Neuroinflammation}

Through a combination of proteomic,metabolomic, transcriptomic, and perturbation studies, we found thatsphingolipid metabolism in astrocytes triggers the interaction of the $\mathrm{C} 2$ domainin cytosolic phospholipase A2 (cPLA2) with the CARD domain in mitochondrialantiviral signaling protein (MAVS), boosting NF- $\kappa \mathrm{B}$-driven transcriptionalprograms that promote CNS inflammation in experimental autoimmuneencephalomyelitis (EAE) and, potentially, multiple sclerosis. cPLA2 recruitmentto MAVS also disrupts MAVS-hexokinase 2 (HK2) interactions, decreasing HKenzymatic activity and the production of lactate involved in the metabolicsupport of neurons. Miglustat, a drug used to treat Gaucher and NiemannPickdisease, suppresses astrocyte pathogenic activities and ameliorates EAE[12].In neurodegenerative diseases, debris of dead neurons are thought to triggerglia-mediated neuroinflammation, thus increasingneuronal death. The neurotoxic proteins associated with these diseases are expressed in Microglia enough to directly trigger the death of naive neurons, which are transmitted by activating the naive astrocyte to the A1 state. To a large extent, transmission of injury occurs by the release of Mitochondria into the neuronal environment. The number of damaged Mitochondria relative to Functional Mitochondria released by Microglia, and the resulting neuronal damage, is determined by apoptosis mediated mitochondrial breakdown within the Neuroglia. Increased inflammation and neuronal cell death by Mitochondria with extracellular dysfunction suggest a new intervention for neurodegeneration -- inhibition of Mitochondrial Microglia, this prevents dysfunctional Mitochondria from entering the brain's extracellular environment without affecting the release of healthy neuroprotective Mitochondria[13].Themicroglial receptors CD33 and TREM2 have been associated with risk forAlzheimer's disease (AD). It showed that knockout of CD33 attenuated amyloid beta $(A \beta)$ pathologyand improved 
cognition in 5xFAD mice, both of which were abrogated byadditional TREM 2 knockout. Knocking out TREM 2 in 5xFAD mice exacerbated A $\beta$ pathology and neurodegeneration but reduced Iba1+ cell numbers, allof which could not be rescued by additional CD33 knockout[14].

\section{Conclusion}

In summary, inhibition of neuroinflammation is an important factor in the treatment of neurological disorder and maintenance of normal brain function, and the DA receptor is an important drug target for the treatment of brain diseases. Dopamine can inhibit neuroinflammation by regulating the immune process and then inhibiting the release of proinflammatory mediators. In many inflammatory conditions, common diseases such as Osteoarthritis, rheumatoid arthritis and, most recently, COVID-19, major complications and extensive tissue damage occur when the immune system is excessively and uncontrollably activated. Finding new ways to selectively control such overactivity may have significant clinical benefits. Alzheimer's Disease (AD) and Parkinson's disease (PD) are both strongly linked to neuroinflammation in the Neurodegeneration. Neuroinflammation mediated by Microglia (MG) is a major factor in both diseases. Current treatments for PD are only temporarily effective and can only be used in a small number of patients. These therapies can neither restore missing or degraded dopaminergic neurons nor delay or halt disease progression, so it is critical to develop more effective treatment strategies. neuroinflammation has been found to lead to the degeneration of dopaminergic neurons, so it is important to target neuroinflammation to develop new therapeutic strategies to slow the progression of Neurodegeneration. At present, the use of traditional Chinese medicine for the treatment of neuroinflammatory diseases has gradually become a research hotspot.

\section{References}

1. Alessandro Villa,Elisabetta Vegeto,Angelo Poletti,et al.Estrogens, Neuroinflammation, and Neurodegeneration[J].Endocr Rev.,2016,37(4):372402.

2. I.Léna,S.Bradshaw,J.Pintar,et al.Adaptive changes in the expression of central opioid receptors in mice lacking the dopamine D2 receptor gene[J].Neuroscience,2008,153(3): 773-788.

3. Zhang Shuzhen, Shao Wei, Zhou Jiawei. Astrocyte expression of dopamine D2 receptor inhibits neuroinflammation. Journal of Cell Biology, 2013,35(2) : 119-122.

4. Pablo Trindade,Erick Correia Loiola,Juciano Gasparotto,et al, Short and long TNF-alpha exposure recapitulates canonical astrogliosis events in humaninduced pluripotent stem cells-derived astrocytes[J]. Glia,2020, 68 (7) :1396-1409.
5. Li QF, Hao H, Tu WS, et al. Maresins: antiinflammatory pro-resolving mediators with therapeutic potential[J].Eur Rev Med Pharmacol Sci.,2020,24(13):7442-7453.

6. Chen Cong, Wu fan, Qin Maoxin,et al. Research progress in the mechanism of immune inflammatory response and clinical intervention after severe trauma[J].Chinese Journal of Trauma, 2019,35(10) : 953-960.

7. Meng Rui, Liu Yunxia. Progress in pharmacological studies of Dopamine receptor agonists $[\mathrm{J}]$. For Medicine, January 1,2013:357.

8. Yang Xuan, Wang Yubo, Zhang Yun,et al. Function and regulation of DRD2 in Midbrain dopaminergic neurons [J] . Frontiers of medicine, 2019,48(3) : 1619.

9. Jang suk-jung. The role of glial dopamine D2 receptor in the brain innate immune regulation[D] . University of Chinese Academy of Sciences, 2013.

10. Tang Mi. Dopamine Receptor D2 modulates $\alpha \beta$ crystallin and inhibits neuroinflammation [D] . Nanjing Medical University, 2012.

11. Yuqing $\mathrm{Wu}$, Yingchao $\mathrm{Hu}$, Bingwei Wang ,et al.Dopamine Uses the DRD5-ARRB2-PP2A Signaling Axis to Block the TRAF6-Mediated NF- $\kappa$ B Pathway and Suppress Systemic Inflammation[J].Molecular Cell,2020, 78(1):42-58.

12. Chao C, Gutiérrez Vázquez C, Rothhammer V, et al.Metabolic Control of Astrocyte Pathogenic Activity via cPLA2-MAVS[J]. Cell,2019,179(7):1483-1498.

13. Joshi A.U., Minhas P.S., Liddelow S.A., et al (2019). Fragmented mitochondria released frommicroglia trigger A1 astrocytic response and propagate inflammatoryneurodegeneration[J].Nat Neurosci. ,2019,22(10):1635-1648.

14. Griciuc A, Patel S, Federico AN, et al. TREM2 Acts Downstream of CD33 in Modulating Microglial Pathologyin Alzheimer's Disease[J].Neuron.,2019,103(5):820-835. 